Research Article

\title{
Fast Phased Array Calibration by Power-Only Measurements Twice for Each Antenna Element
}

\author{
Guolong He (iD, Xin Gao, and Hui Zhou \\ Beijing Institute of Tracking and Telecommunication Technology, Beijing 100094, China \\ Correspondence should be addressed to Guolong He; heguolong@alumni.sjtu.edu.cn
}

Received 1 October 2018; Revised 23 February 2019; Accepted 10 March 2019; Published 17 April 2019

Academic Editor: Chien-Jen Wang

Copyright (C) 2019 Guolong He et al. This is an open access article distributed under the Creative Commons Attribution License, which permits unrestricted use, distribution, and reproduction in any medium, provided the original work is properly cited.

\begin{abstract}
This paper proposes a novel power-only measurement method for phased array antenna calibration. Besides the total array power, only one phase shift and two power measurements for each array element are required to determine the element complex electric field distortion, one by shifting the element's phase of $\pi / 2$ and the other by turning the element under test off. The theory of the proposed calibration method is given, and the closed form formulation of the element amplitude and phase distortions is derived. From the mathematical point of view, it is the minimum required measurements that use two scalar values to determine one complex vector. Numerical simulations and experiments are conducted to validate and demonstrate the effectiveness of the proposed calibration method.
\end{abstract}

\section{Introduction}

Phased array antenna plays a very important role in modern radar and wireless communication systems [1-9]. Due to some inevitable errors and uncertainties, such as mechanical manufacturing tolerance, temperature variation, and electronic and radiofrequency component aging, the realistic array element excitation (both amplitude and phase) deviates from their ideal values. This would cause array radiation pattern distortion and degrade the overall performance, which must be carefully considered for practical system design. Hence, to maintain an acceptable performance of phased array antenna, calibrations are always necessary by measuring the excitation distortions of individual array elements and compensating them as accurate as possible before and/or during their operation.

A variety of calibration techniques have been proposed so far for phased array antenna calibration [10-23]. In [10], Silverstein proposed a method which controls the element phases based on time-multiplexed orthogonal codes; thus, the individual element excitation can be derived from the combined array signal. Some other phase toggling methods change the element's phase shift to gather the required element excitation information [11-14]. Lee et al. [11] switched the element's phase between the two states of 0 and $\pi$ and determined the element excitation by comparing the combined array signal at these two states. For all the abovementioned methods, they require coherent measurement between the calibration source and sink to obtain the complex value containing both amplitude and phase information. It is not always convenient to apply them for operating phased array system, especially for far-field calibration scenario with a long distance between the source and sink or in the millimeter wave band where the phase is sensitive and can be measured only with a restricted accuracy.

Compared to these complex measurement methods, some other methods which involve amplitude- (or power) only measurements are considered to be more robust and convenient. In such case, coherent measurements are no longer required with precise synchronization between the transmitter and receiver. Many efforts have also been made that follow this principle. He et al. [15] proposed a method by periodically modulating the element phase sequentially and then applying a fast Fourier transform (FFT) analysis; the element complex electric field excitation is obtained from the corresponding modulation frequency harmonics of the combined array signal. The rotating element electric field vector (REV) method is another well-known power-only 


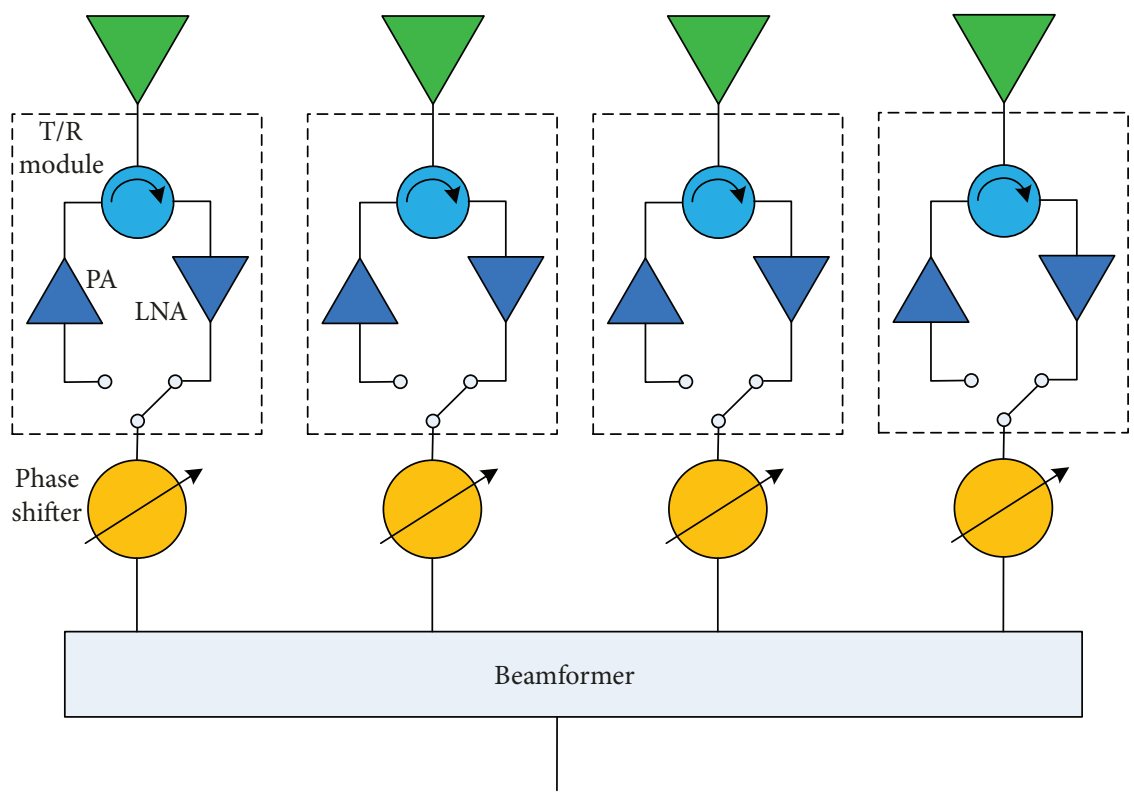

To transmitter/receiver

Figure 1: Typical architecture of an active phased antenna array.

measurement method; it measures the amplitude of the array by shifting the element phase from 0 to $2 \pi$ continuously $[16,17]$. The element complex electric field can be determined from statistical signal processing of the three parameters, the maximum and minimum power of the array signal, and the element rotation phase to maximum power. Although it is very simple and easy to use, however, a large amount of measurements for each element is needed in the conventional REV method. In 2001, Sorace [18] pointed out that measurement results at four orthogonal phase shifts are sufficient enough to obtain a maximum likelihood estimation for each array element. Long et al. [19] also derived an expression that requires two phase shifts of $\pi / 2$ and $\pi$ to yield the element complex excitation information. All these above efforts are made to reduce the amount of required measurements and overcome the inefficiency of the original REV method.

In this paper, we propose a novel power-only measurement method to calibrate the element complex excitation distortion. For each array element, two power measurements are enough to determine the element complex electric field, one by shifting the element's phase of $\pi / 2$ and the other by turning the element under test off. This method only needs some simple mathematical calculation but requires no auxiliary calibration element or any additional hardware. From the mathematical point of view, it is the minimum required measurements that use two scalar values to determine one complex vector. The rest of this paper is organized as follows. In Section 2, the proposed calibration method is introduced, and the closed-form formulation for element amplitude and phase distortions is derived. Then, in Section 3 , the calibration accuracy is analyzed mathematically; the standard derivations of both the amplitude and phase estimations are obtained. Section 4 uses an eight-element patch antenna array to demonstrate the calibration procedure and investigate its performance. In Section 4, a four-element dipole antenna array is fabricated and tested to further validate the effectiveness of the proposed method. Finally, this paper is concluded in Section 5.

\section{Methodology}

Figure 1 illustrates a typical configuration for $N$-element active phased antenna array. It contains both transmitting and receiving modes by adopting the T/R module. Each antenna element has a phase shift for electronic beam scanning. The method proposed in this paper can be used in not only the transmitting mode but also the receiving mode. Let $E_{n}$ and $\varphi_{n}$ denote the amplitude and phase of the $n$th antenna element, $n=1,2, \ldots N$. The phased array electric field $\mathbf{E}$, the combination of the individual element electric fields, can be described as follows:

$$
\mathbf{E}=E_{0} \exp \left(j \phi_{0}\right)=\sum_{n=1}^{N} \mathbf{E}_{n}=\sum_{n=1}^{N} E_{n} \exp \left(j \phi_{n}\right)
$$

where $E_{0}$ and $\phi_{0}$ are the amplitude and phase of the array electric field, respectively. As shown in Figure 2, in order to calibrate the $n$th array element complex excitation distortion, we can divide equation (1) into two parts:

$$
\begin{aligned}
\mathbf{E}_{0} & =\mathbf{E}_{\tilde{n}}+\mathbf{E}_{n}=E_{\tilde{n}} \exp \left(j \phi_{\tilde{n}}\right)+E_{n} \exp \left(j \phi_{n}\right) \\
& =\left(E_{\tilde{n}} \cos \phi_{\tilde{n}}+E_{n} \cos \phi_{n}\right)+j\left(E_{\tilde{n}} \sin \phi_{\tilde{n}}+E_{n} \sin \phi_{n}\right),
\end{aligned}
$$

where $\mathbf{E}_{\tilde{n}}=E_{\tilde{n}} \exp \left(j \phi_{\tilde{n}}\right)$ denotes the combined electric field of the array expecting the $n$th element, and it has the power 


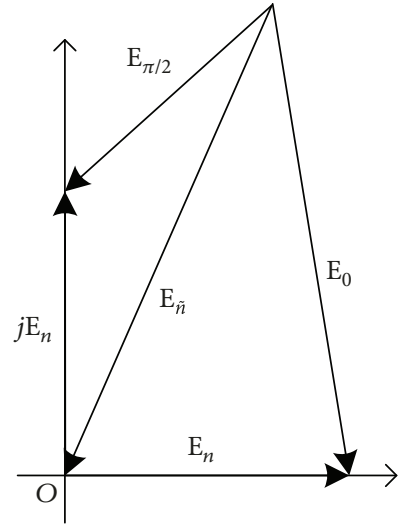

FIGURE 2: Vector representation of the proposed calibration method.

of $P_{\tilde{n}}=E_{\tilde{n}}^{2}$ which can be easily measured by turning the $n$th element under test off or terminating it with a matched load.

From equation (2), the array power can be expressed as

$$
\begin{aligned}
P_{0} & =E_{0}^{2}=\left(E_{\tilde{n}} \cos \phi_{\tilde{n}}+E_{n} \cos \phi_{n}\right)^{2}+\left(E_{\tilde{n}} \sin \phi_{\tilde{n}}+E_{n} \sin \phi_{n}\right)^{2} \\
& =E_{\tilde{n}}^{2}+E_{n}^{2}+2 E_{\tilde{n}} E_{n} \cos \left(\phi_{\tilde{n}}-\phi_{n}\right) .
\end{aligned}
$$

We denote the phase difference $\varphi_{n}=\phi_{\tilde{n}}-\phi_{n}$; thus, equation (3) can be further rewritten as

$$
\begin{aligned}
P_{0} & =E_{\tilde{n}}^{2}+E_{n}^{2} \cos ^{2} \varphi_{n}+E_{n}^{2} \sin ^{2} \varphi_{n}+2 E_{\tilde{n}} E_{n} \cos \varphi_{n} \\
& =\left(E_{\tilde{n}}+E_{n} \cos \varphi_{n}\right)^{2}+\left(E_{n} \sin \varphi_{n}\right)^{2},
\end{aligned}
$$

where $E_{n} \cos \varphi_{n}$ and $E_{n} \sin \varphi_{n}$ are the real and imaginary parts of the $n$th array element complex excitation, respectively.

We then shift the phase of the $n$th antenna element by $\pi / 2$; then, the corresponding electric filed $\mathbf{E}_{\pi / 2}$ and the array power $P_{\pi / 2}$ can be written as

$$
\begin{aligned}
\mathbf{E}_{\pi / 2} & =E_{\tilde{n}} \exp \left(j \phi_{\tilde{n}}\right)+E_{n} \exp \left(j \phi_{n}\right) \exp (j \pi / 2) \\
& =\left(E_{\tilde{n}} \cos \phi_{\tilde{n}}-E_{n} \sin \phi_{n}\right)+j\left(E_{\tilde{n}} \sin \phi_{\tilde{n}}+E_{n} \cos \phi_{n}\right),
\end{aligned}
$$

$$
\begin{aligned}
P_{\pi / 2} & =\left(E_{\tilde{n}} \cos \phi_{\tilde{n}}-E_{n} \sin \phi_{n}\right)^{2}+\left(E_{\tilde{n}} \sin \phi_{\tilde{n}}+E_{n} \cos \phi_{n}\right)^{2} \\
& =E_{\tilde{n}}^{2}+E_{n}^{2}+2 E_{\tilde{n}} E_{n} \sin \left(\phi_{\tilde{n}}-\phi_{n}\right) \\
& =E_{\tilde{n}}^{2}+E_{n}^{2} \cos ^{2} \varphi_{n}+E_{n}^{2} \sin ^{2} \varphi_{n}+2 E_{\tilde{n}} E_{n} \sin \varphi_{n} \\
& =\left(E_{\tilde{n}}+E_{n} \sin \varphi_{n}\right)^{2}+\left(E_{n} \cos \varphi_{n}\right)^{2}
\end{aligned}
$$

From equations (4) and (6), the two unknowns $E_{n} \cos \varphi_{n}$ and $E_{n} \sin \varphi_{n}$ can be derived as follows:

$$
\left\{\begin{array}{l}
E_{n} \cos \varphi_{n}=\frac{\left(-2 P_{\tilde{n}}+P_{0}-P_{\pi / 2}+Q\right)}{4 \sqrt{P_{\tilde{n}}}}, \\
E_{n} \sin \varphi_{n}=\frac{\left(-2 P_{\tilde{n}}-P_{0}+P_{\pi / 2}+Q\right)}{4 \sqrt{P_{\tilde{n}}}},
\end{array}\right.
$$

or

$$
\left\{\begin{array}{l}
E_{n} \cos \varphi_{n}=\frac{\left(-2 P_{\tilde{n}}+P_{0}-P_{\pi / 2}-Q\right)}{4 \sqrt{P_{\tilde{n}}}}, \\
E_{n} \sin \varphi_{n}=\frac{\left(-2 P_{\tilde{n}}-P_{0}+P_{\pi / 2}-Q\right)}{4 \sqrt{P_{\tilde{n}}}},
\end{array}\right.
$$

where

$$
Q=\sqrt{4 P_{\tilde{n}} P_{0}+4 P_{\tilde{n}} P_{\pi / 2}+2 P_{0} P_{\pi / 2}-4 P_{\tilde{n}}^{2}-P_{0}^{2}-P_{\pi / 2}^{2}} .
$$

Since $P_{0}$ is the same for all the elements, thus, only two additional power-only measurements $P_{\tilde{n}}$ and $P_{\pi / 2}$ are required to calculate each element excitation distortion. Like the conventional REV method, it also has two possible solutions in equations (7a) and (7b). This ambiguity can be easily eliminated if the initial phases of the individual elements are set to make $E_{\tilde{n}}>E_{n}$ [19]. From equations (7a) and $(7 \mathrm{~b})$, the relative amplitude and phase distortion, $k_{n}$ and $x_{n}$, of the $n$th antenna element compared to the array signal can be derived as

$$
k_{n} \exp \left(-j \chi_{n}\right)=\frac{\mathbf{E}_{0}}{\mathbf{E}_{n}}=\frac{\mathbf{E}_{\tilde{n}}+\mathbf{E}_{n}}{\mathbf{E}_{n}}=\frac{E_{\tilde{n}}}{E_{n}} \exp \left(j \varphi_{n}\right)+1,
$$

where

$$
\begin{aligned}
& E_{n}=\sqrt{\left(E_{n} \cos \varphi_{n}\right)^{2}+\left(E_{n} \sin \varphi_{n}\right)^{2},} \\
& \varphi_{n}=\tan ^{-1}\left(E_{n} \sin \varphi_{n} / E_{n} \cos \varphi_{n}\right) .
\end{aligned}
$$

\section{Calibration Accuracy Analysis}

Recall that $E_{n} \cos \varphi_{n}$ and $E_{n} \sin \varphi_{n}$ are the real and imaginary parts of the $n$th array element complex excitation, respectively. Since these two components are orthogonal to each other, they can be modeled as independent Gaussian distributions with zero mean and the variance $1 /(2 \times \mathrm{SNR})$, where SNR is the signal-to-noise ratio of the power measurement. By squaring them and adding together as in equation (10a), the result of $P_{n}$ should have doubly noncentral chi-squared distribution with two degrees of freedom. From the above derivation, the amplitude estimation $E_{n}$, as the square root of $P_{n}$, should have a Rayleigh distribution, and it has the standard deviation as [14]

$$
\sigma\left(E_{n}\right)=\frac{\sqrt{2 \mathrm{SNR}+1}}{\mathrm{SNR}}
$$

The phase estimation $\varphi_{n}$, as shown in equation (10b), can be viewed as the ratio of two Gaussian distributions. 


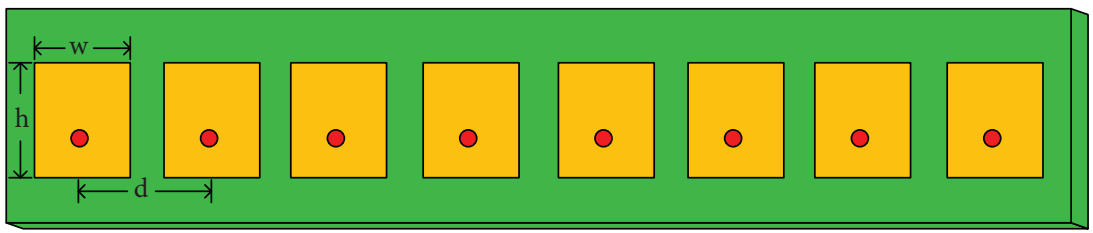

FIGURE 3: Geometry of the eight-element linear patch antenna array.

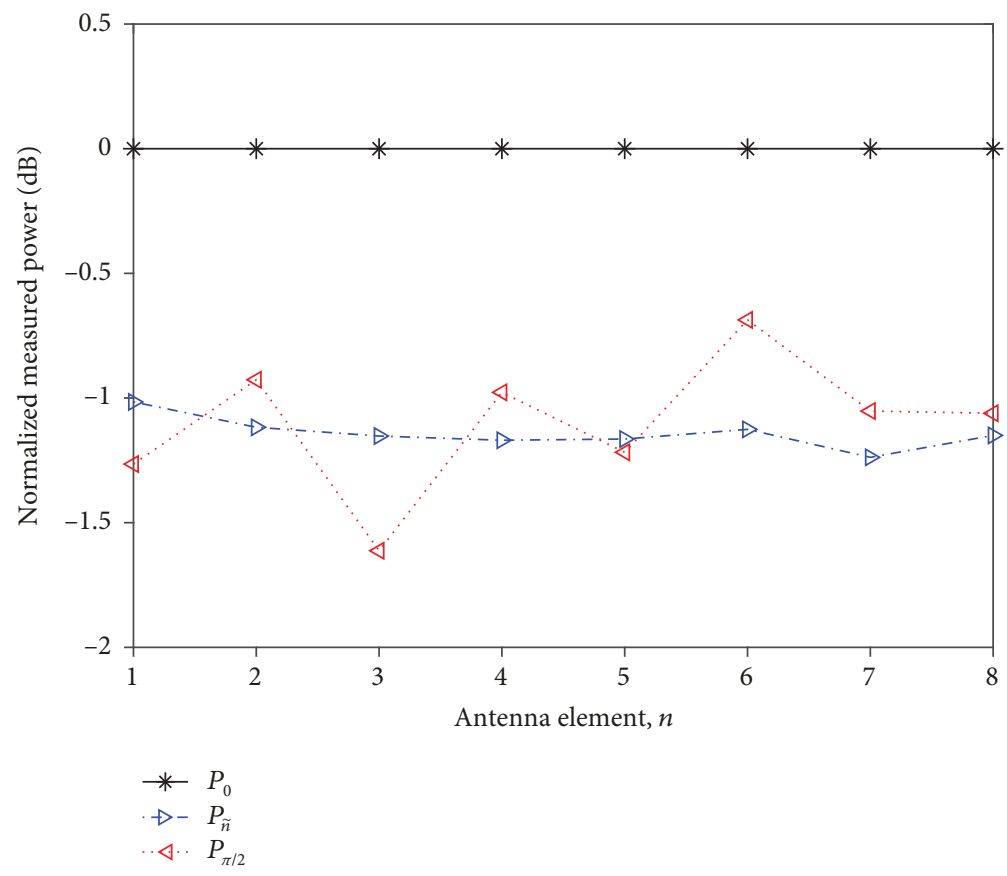

FIGURE 4: The normalized power measurement results.

Its probability density function (PDF) can be expressed as follows [24]:

$$
\begin{aligned}
p(y)= & \frac{1}{\pi\left(y^{2}+1\right) \exp (\mathrm{SNR})}+\frac{\sqrt{\mathrm{SNR}}}{\sqrt{\pi}\left(y^{2}+1\right)^{3 / 2}} \\
& \times \exp \left(-\frac{\mathrm{SNR} \cdot y^{2}}{y^{2}+1}\right) \times \operatorname{erf}\left(\sqrt{\frac{\mathrm{SNR}}{y^{2}+1}}\right),
\end{aligned}
$$

where $y=\tan \varphi_{n}$ also has approximate Gaussian distribution for high SNR. Finally, the standard deviation of the phase estimation in degree is approximated as [14]

$$
\sigma\left(\varphi_{n}\right) \approx \frac{180}{\pi \sqrt{2 \mathrm{SNR}}} .
$$

\section{Numerical Simulations}

In order to verify the effectiveness of the proposed method, an eight-element linear patch antenna array working at S-band is numerically simulated to demonstrate the calibration procedure and evaluate its performance. The phased array contains eight half-wavelength spaced patch antenna elements. As shown in Figure 3, the rectangular patch antennas are with length $h=4 \mathrm{~cm}$ and width $w=$ $3 \mathrm{~cm}$ on the lossless substrate, which has the dielectric constant $\varepsilon_{r}=2.2$ and thickness of $1.57 \mathrm{~mm}$. The feed is offset by $5.5 \mathrm{~mm}$ from the center of the rectangular patch antenna.

During the simulation, random initial amplitude and phase excitation are preset for each antenna element. The standard deviation for element amplitude and phase error is set to $0.5 \mathrm{~dB}$ and $10 \mathrm{deg}$, respectively. The power measurement is made at the far-field region of the phased array, and the measurement SNR is set as $20 \mathrm{~dB}$. Figure 4 illustrates one example of the simulated far-field power measurement results. The black asterisks denote the normalized combined array power $P_{0}$, which is normalized to $0 \mathrm{~dB}$. The red and blue triangles denote the two power measurements $P_{\tilde{n}}$ and $P_{\pi / 2}$ for each array element. They vary from each other since every element has different amplitudes and phase distortions. Figures 5 and 6 illustrate the calibration results for each element derived from the proposed method. Compared to the preset values, the corresponding calibration errors are $0.26 \mathrm{~dB}$ and $2.17 \mathrm{deg}$ for amplitude and phase distortion, respectively. It can be seen that the estimation results have very good coincidence with the preset values. The 


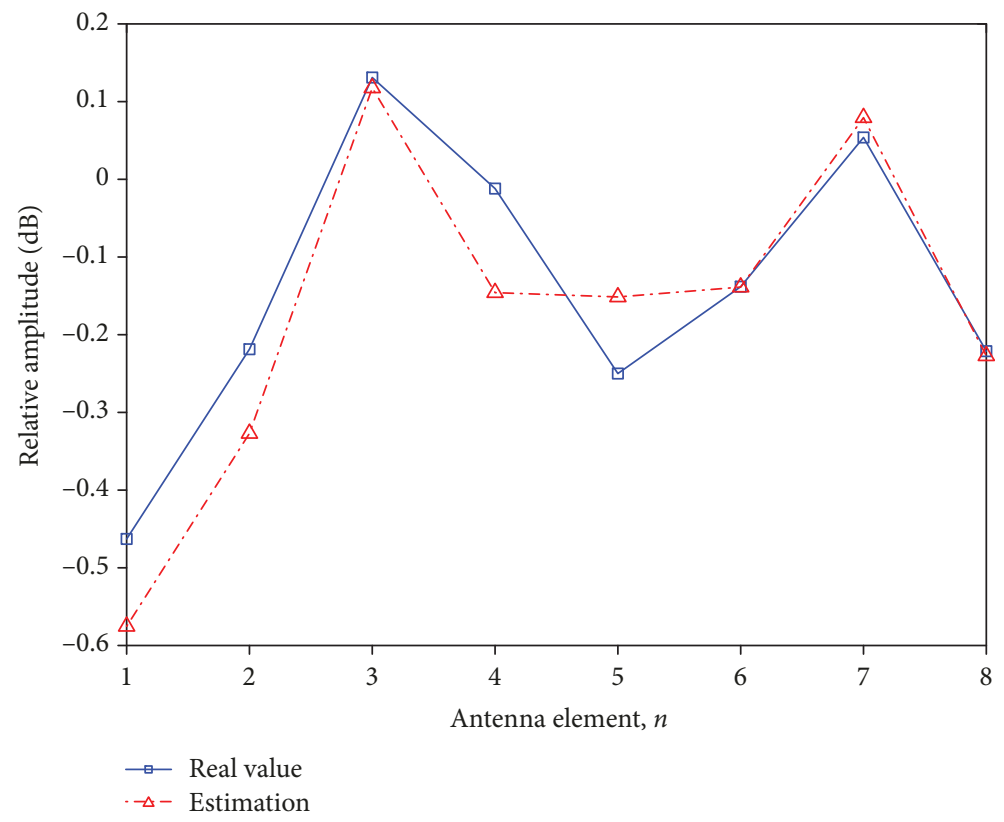

FIgURE 5: The array element relative amplitude and the corresponding estimation results.

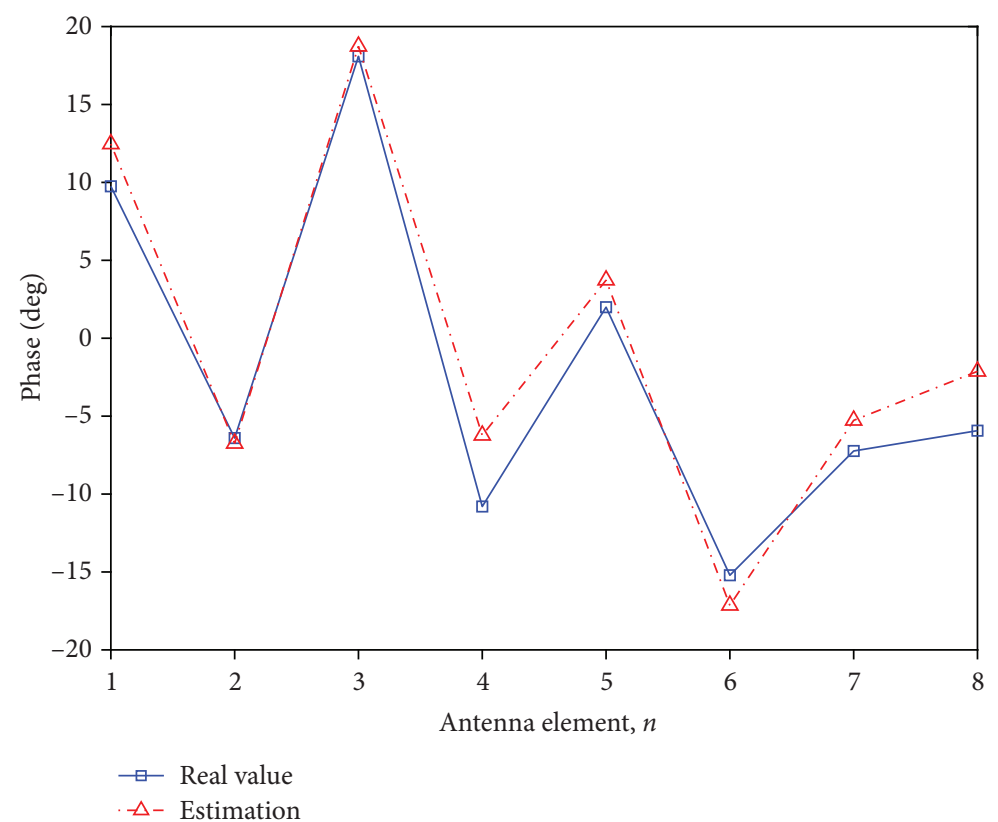

Figure 6: The array element relative phase and the corresponding estimation results.

investigation is further conducted by 1000 Monte Carlo simulations. The histogram of element amplitude and phase calibration errors is presented in Figures 7 and 8, both of which show nearly normal distributions. Moreover, approximately $84 \%$ of the amplitude calibration errors fall in the region of $-1 \mathrm{~dB}$ and $+1 \mathrm{~dB}$, while the probability percentage of phase calibration error in the range of $-5 \mathrm{deg}$ to $+5 \mathrm{deg}$ is nearly $78 \%$. The standard deviations of element amplitude and phase estimation errors for different SNR conditions are presented in Figures 9 and 10. It can be seen from the results that the mean square error (MSE) of both amplitude and phase calibration decreases dramatically with the increase of the SNR. For SNR larger than $30 \mathrm{~dB}$ (this condition can be easily achieved in practice), the amplitude and phased calibration error are less than $0.05 \mathrm{~dB}$ and $0.5 \mathrm{deg}$; such high accuracy exceeds most phased array calibration demands. 


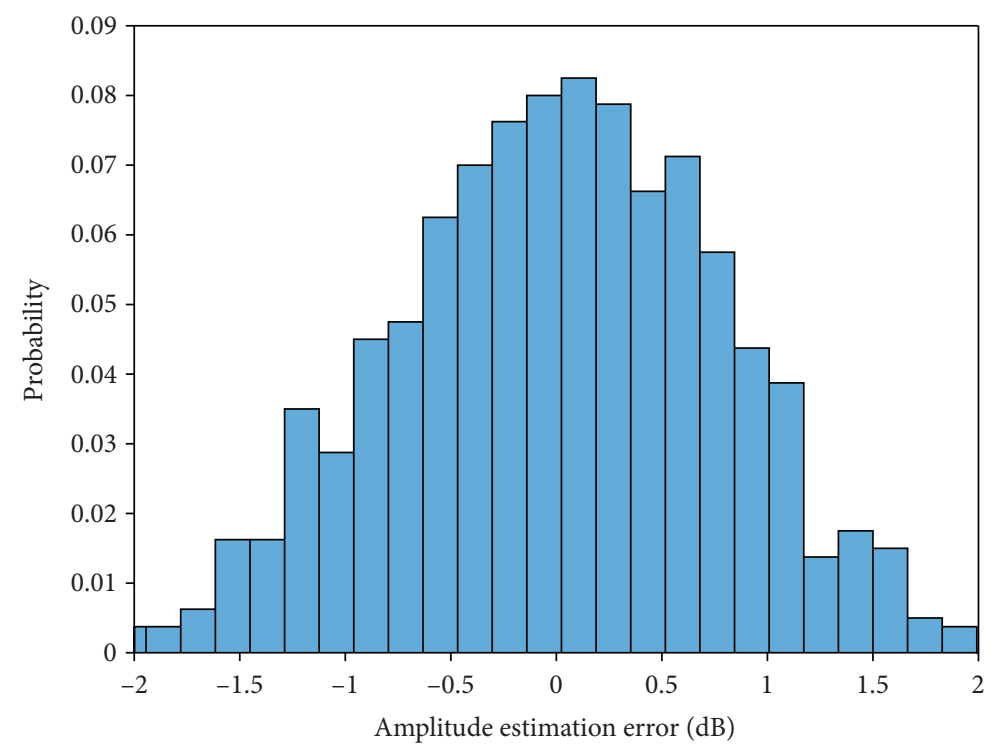

FIgURE 7: The amplitude calibration error histogram by 1000 Monte Carlo simulations.

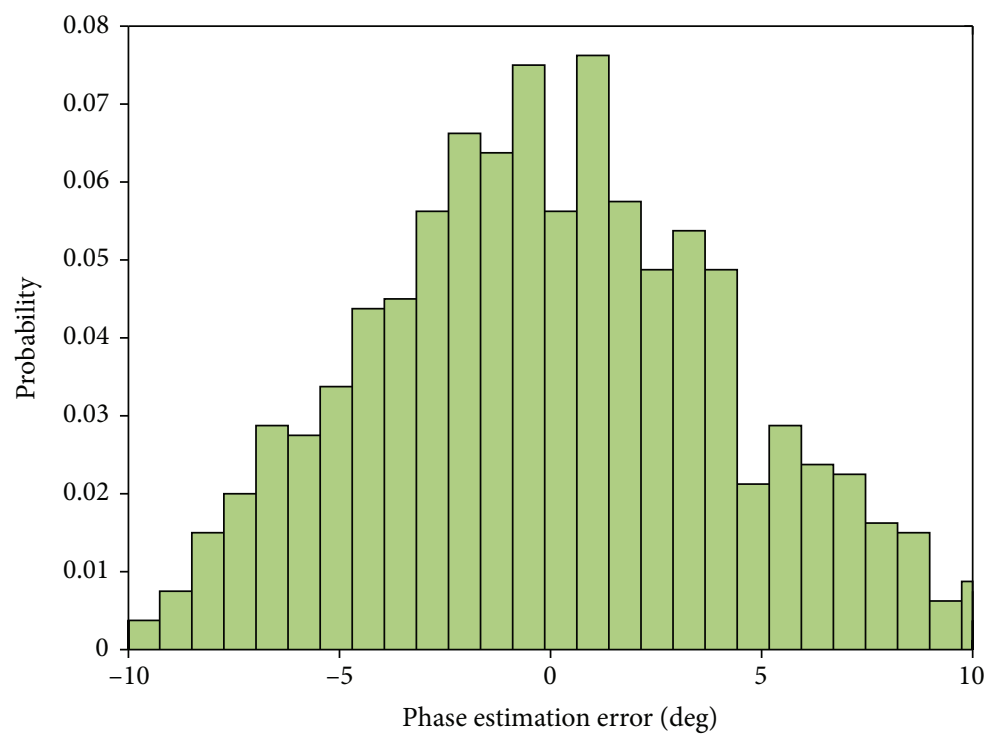

Figure 8: The phase calibration error histogram by 1000 Monte Carlo simulations.

\section{Experiment Demonstration}

To further validate the effectiveness of the proposed method, a four-element dipole antenna phased array is fabricated and tested. As shown in Figure 11, this antenna array works at S-band with full transmitting and receiving duplex function. For the transmitting mode calibration, a pure sinusoidal wave source is fed to the phased antenna array, the signal goes to each antenna element from the digital beamforming network (DBF) then transmits to the free space, and a calibration receiver is placed at the far field of the phased array and connected with a power meter or spectrum analyzer, while for the receiving mode calibration, the calibration transmitter at the far-field region transmits a pure sinusoidal wave, and the phased array receives the plane wave by the elements and the combined array signal is connected to a power meter. follows:

The phased array calibration process is described as

(1) First, each antenna element is measured individually using a vertical network analyzer (VNA), and their amplitude and phase distortions are obtained

(2) The combined power of the test array $P_{0}$ is measured by a power meter

(3) By setting the DBF weight of the $n$th antenna element to zero sequentially, the combined power of the 


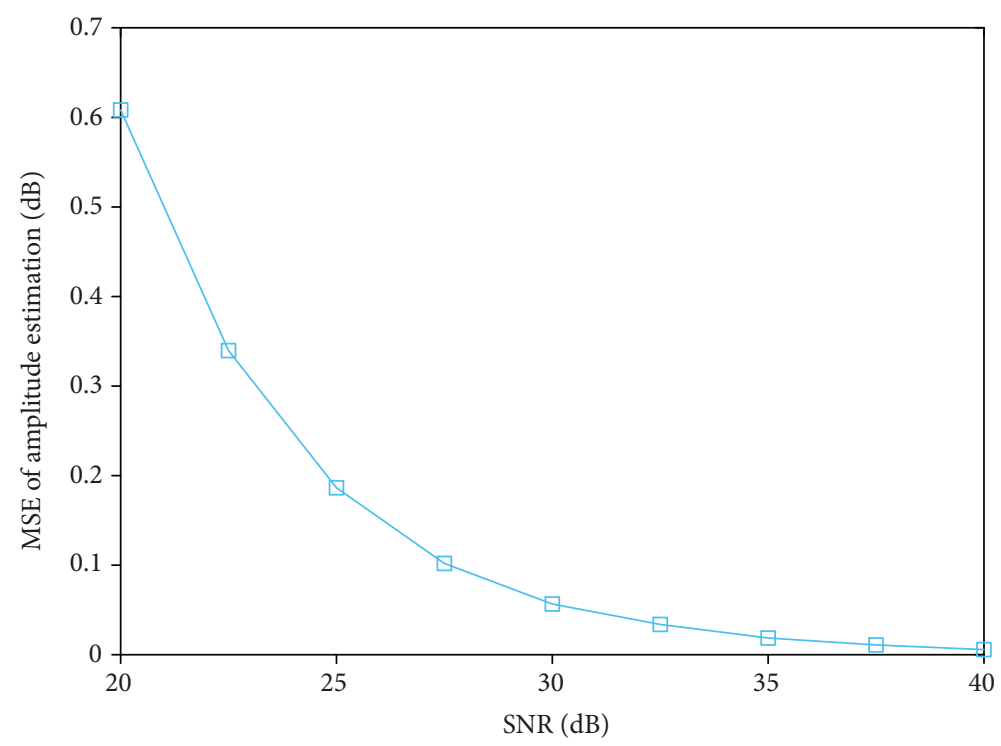

FIgURE 9: The amplitude calibration error under different SNR conditions.

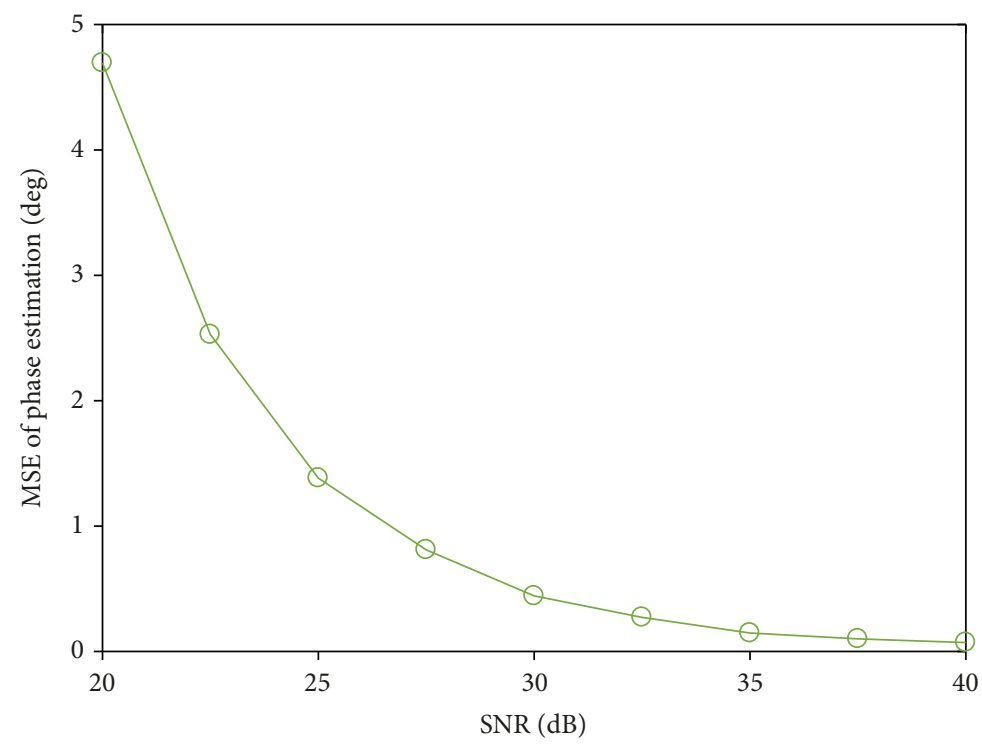

FIGURE 10: The phase calibration error under different SNR conditions.

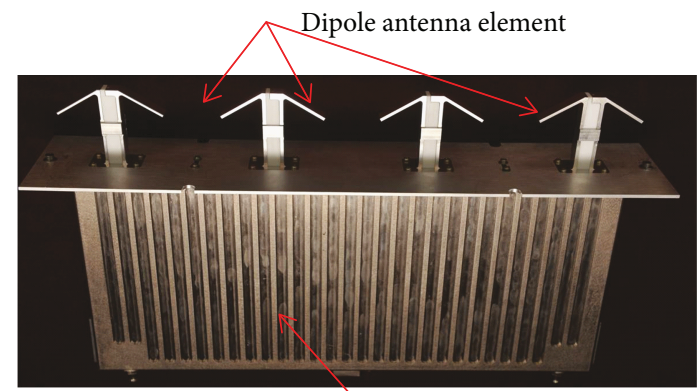

Integrated T/R module \& DBF

FIGURE 11: The four-element dipole antenna array for experiment demonstration. array expecting the $n$th element $P_{\tilde{n}}$ is measured by a power meter

(4) By setting the phase of the $n$th antenna element to $\pi / 2$ sequentially, the combined power of the array $P_{\pi / 2}$ is measured by a power meter

(5) The amplitude and phase calibration results are calculated by using equation (9) and compared with the results from step (1)

Both the transmitting and receiving modes are carefully calibrated in our work. The results of the receiving mode are presented in Table 1, while the results of the transmitting mode are similar and are not given here for the constraint of 
TABLE 1: Calibration results of the four-element patch antenna array.

\begin{tabular}{|c|c|c|c|c|c|}
\hline Antenna element & & $n=1$ & $n=2$ & $n=3$ & $n=4$ \\
\hline \multirow{3}{*}{ Power measurement results } & $P_{0}(\mathrm{dBm})$ & \multicolumn{4}{|c|}{-39.2} \\
\hline & $P_{\tilde{n^{\sim}}}(\mathrm{dBm})$ & -41.7 & -41.7 & -41.7 & -41.9 \\
\hline & $P_{\pi / 2}(\mathrm{dBm})$ & -41.6 & -41.2 & -41.3 & -41.3 \\
\hline \multirow{2}{*}{ Calibration } & $k_{n}(\mathrm{~dB})$ & 0 & -0.057 & -0.057 & 0.517 \\
\hline & $x_{n}(\operatorname{deg})$ & 0 & 6.42 & 4.78 & 6.61 \\
\hline \multirow{2}{*}{ VNA results } & $k_{n}(\mathrm{~dB})$ & 0 & -0.1 & -0.2 & 0.5 \\
\hline & $x_{n}(\mathrm{deg})$ & 0 & 7 & 4.7 & 6.3 \\
\hline
\end{tabular}

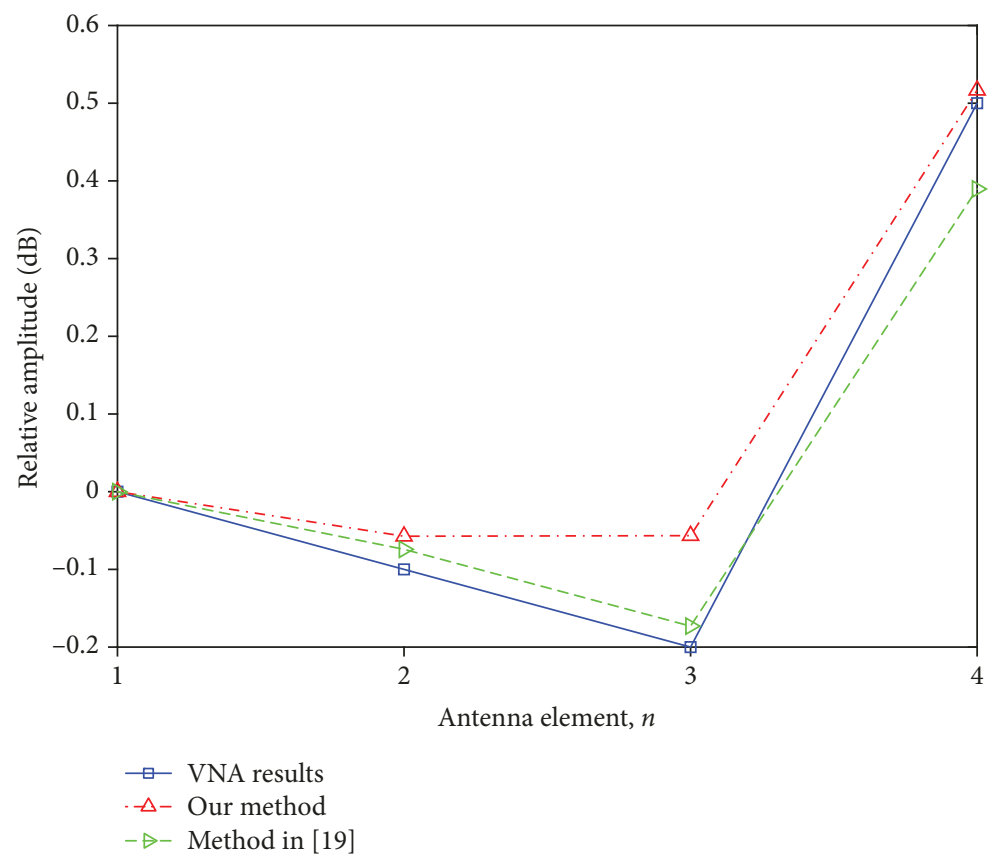

Figure 12: The amplitude calibration results of the four-element patch antenna array.

the paper length. During the experiments, the power measurements are accomplished by using Agilent E4405B Spectrum Analyzer, while Keysight M9374A PXIe Vector Network Analyzer is used for single-antenna element calibration. The reading accuracy of the power and phase measurements is set as $0.1 \mathrm{~dB}$ and $0.1 \mathrm{deg}$, respectively. Figures 12 and 13 illustrate the calibration results by our method with the results from VNA measurement. The results by using Long et al.'s method in [19] are also presented for comparison. It can be seen that both the amplitude and phase calibration results by our method have good agreement with the measurement results from VNA and the results from Long et al.'s method. The corresponding standard deviations are $0.06 \mathrm{~dB}$ for amplitude calibration and $0.37 \mathrm{deg}$ for phase calibration.

\section{Conclusion}

Phased array element excitation (both amplitude and phase) always deviates from the ideal values that would cause array performance degradation, which must be carefully calibrated and compensated as accurate as possible for practical phased array system design. This paper proposes a novel power measurement method to obtain the excitation distortions of individual array elements. Besides the total array power, only one phase shift and two power measurements for each array element are required to determine the element complex excitation, one by shifting the element's phase of $\pi / 2$ and the other by turning the element under test off. This method only involves some simple mathematical calculation and requires no auxiliary calibration element or any additional hardware. From the mathematical point of view, it is the minimum required measurements that use two scalar values to determine one complex vector. An eight-element linear patch antenna array is numerically simulated to demonstrate the calibration procedure, and the calibration performance is investigated by Monte Carlo simulations. Furthermore, a four-element dipole antenna array is fabricated and tested to validate the effectiveness of the proposed method. 


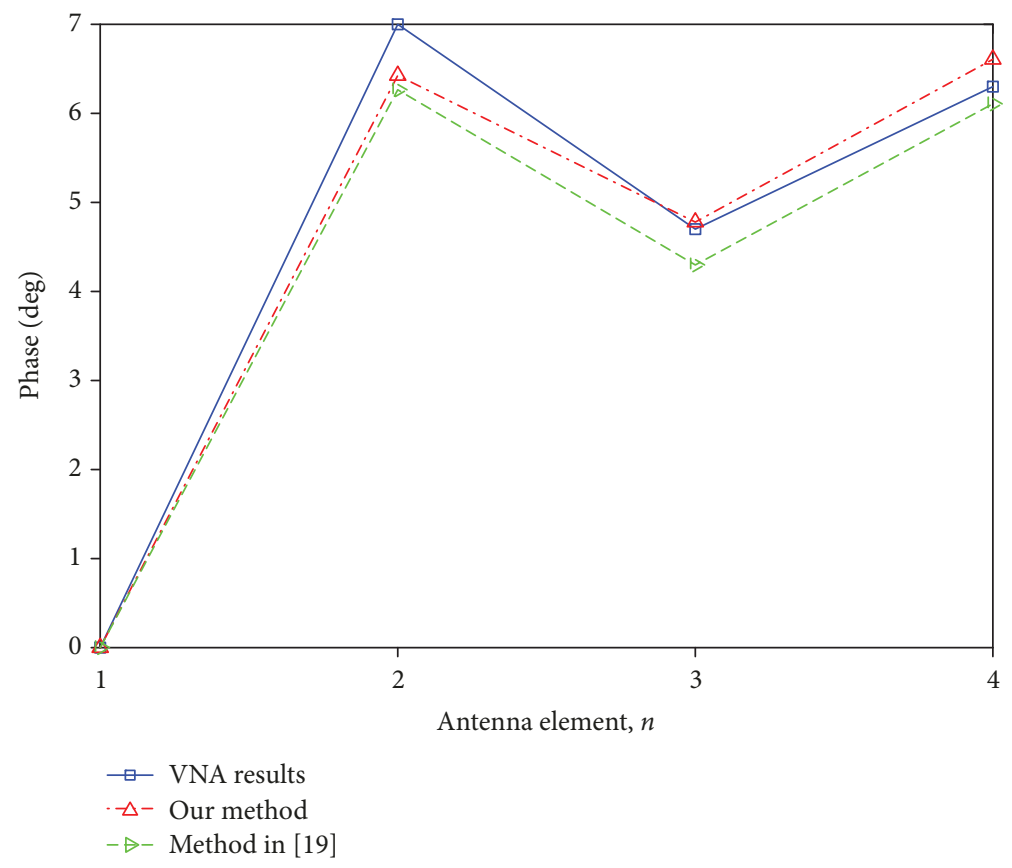

FIGURE 13: The phase calibration results of the four-element patch antenna array.

\section{Data Availability}

The data used to support the findings of this study are available from the corresponding author on reasonable request.

\section{Conflicts of Interest}

The authors declare that there are no conflicts of interest regarding the publication of this paper.

\section{References}

[1] R. S. Elliott, Antenna Theory and Design, Wiley-Interscience, Hoboken, NJ, USA, 2003.

[2] R. J. Mailloux, Phased Array Antenna Handbook, Artech House, Boston, MA, USA, 2005.

[3] R. L. Haupt, Antenna Arrays: a Computational Approach, John Wiley \& Sons, Inc., Hoboken, NJ, USA, 2010.

[4] L. C. Godara, "Applications of antenna arrays to mobile communications. I. Performance improvement, feasibility, and system considerations," Proceedings of the IEEE, vol. 85, no. 7, pp. 1031-1060, 1997.

[5] L. C. Godara, "Application of antenna arrays to mobile communications. II. Beam-forming and direction-of-arrival considerations," Proceedings of the IEEE, vol. 85, no. 8, pp. 1195-1245, 1997.

[6] P. K. Bailleul, "A new era in elemental digital beamforming for spaceborne communications phased arrays," Proceedings of the IEEE, vol. 104, no. 3, pp. 623-632, 2016.

[7] G. He and B. Wu, "Unified particle swarm optimization with random ternary variables and its application to antenna array synthesis," Journal of Electromagnetic Waves and Applications, vol. 28 , no. 6, pp. 752-764, 2014.
[8] M. Thevenot, C. Menudier, A. El Sayed Ahmad et al., "Synthesis of antenna arrays and parasitic antenna arrays with mutual couplings," International Journal of Antennas and Propagation, vol. 2012, Article ID 309728, 22 pages, 2012.

[9] S. Y. Nusenu and A. Basit, "Frequency diverse array antennas: from their origin to their application in wireless communication systems," Journal of Computer Networks and Communications, vol. 2018, Article ID 5815678, 12 pages, 2018.

[10] S. D. Silverstein, "Application of orthogonal codes to the calibration of active phased array antennas for communication satellites," IEEE Transactions on Signal Processing, vol. 45, no. 1, pp. 206-218, 1997.

[11] K. M. Lee, R. S. Chu, and S. C. Liu, "A built-in performance monitoring/fault isolation and correction (PM/FIC) system for active phased-array antennas," IEEE Transactions on Antennas and Propagation, vol. 41, no. 11, pp. 15301540, 1993.

[12] C. N. Hu, "A novel method for calibrating deployed active antenna arrays," IEEE Transactions on Antennas and Propagation, vol. 63, no. 4, pp. 1650-1657, 2015.

[13] G. A. Hampson and A. B. Smolders, "A fast and accurate scheme for calibration of active phased-array antennas," in IEEE Antennas and Propagation Society International Symposium. 1999 Digest. Held in conjunction with: USNC/URSI National Radio Science Meeting (Cat. No.99CH37010), pp. 1040-1043, Orlando, FL, USA, 1999.

[14] Y. Lin, X. Bu, W. Zhao, and S. Wang, "Parallel calibration method for phased array antennas with orthogonal and nonorthogonal codes," in 2015 10th International Conference on Communications and Networking in China (ChinaCom), pp. 883-886, Shanghai, China, 2016.

[15] C. He, X. Liang, J. Geng, and R. Jin, "Parallel calibration method for phased array with harmonic characteristic analysis," IEEE Transactions on Antennas and Propagation, vol. 62, no. 10, pp. 5029-5036, 2014. 
[16] S. Mano and T. Katagi, "A method for measuring amplitude and phase of each radiating element of a phased array antenna," Electronics and Communications in Japan, vol. 65, no. 5, pp. 58-64, 1982.

[17] T. Takahashi, H. Miyashita, Y. Konishi, and S. Makino, "Theoretical study on measurement accuracy of rotating element electric field vector (REV) method," Electronics and Communications in Japan (Part I: Communications), vol. 89, no. 1, pp. 22-33, 2006.

[18] R. Sorace, "Phased array calibration," IEEE Transactions on Antennas and Propagation, vol. 49, no. 4, pp. 517-525, 2001.

[19] R. Long, J. Ouyang, F. Yang, W. Han, and L. Zhou, "Fast amplitude-only measurement method for phased array calibration," IEEE Transactions on Antennas and Propagation, vol. 65, no. 4, pp. 1815-1822, 2017.

[20] R. Long, J. Ouyang, F. Yang, W. Han, and L. Zhou, "Multielement phased array calibration method by solving linear equations," Institute of Electrical and Electronics Engineers, vol. 65, no. 6, pp. 2931-2939, 2017.

[21] G. C. Cardarilli, L. D. Nunzio, R. Fazzolari, M. Re, G. Rufolo, and G. Bernocchi, "Analog chain calibration digital beam forming applications," ARPN Journal of Engineering and Applied Sciences, vol. 13, no. 2, pp. 752-760, 2018.

[22] A. H. Adithya, Y. Huang, and R. J. Weber, "Experimental evaluation of a beamforming array calibration system," in 2014 IEEE Aerospace Conference, pp. 1-7, Big Sky, MT, USA, 2014.

[23] Y. Lin, S. Wang, X. Bu, C. Xing, and J. An, "NOMA-based calibration for large-scale spaceborne antenna arrays," IEEE Transactions on Vehicular Technology, vol. 67, no. 3, pp. 2231-2242, 2018.

[24] A. Jeffrey and D. Zwillinger, Table of Integrals, Series and Products, Academic Press, New York, NY, USA, 7th edition, 2007. 


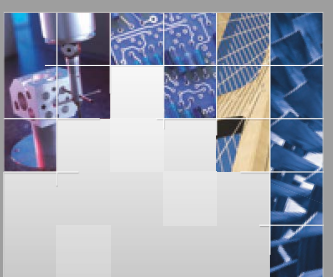

\section{Enfincering}
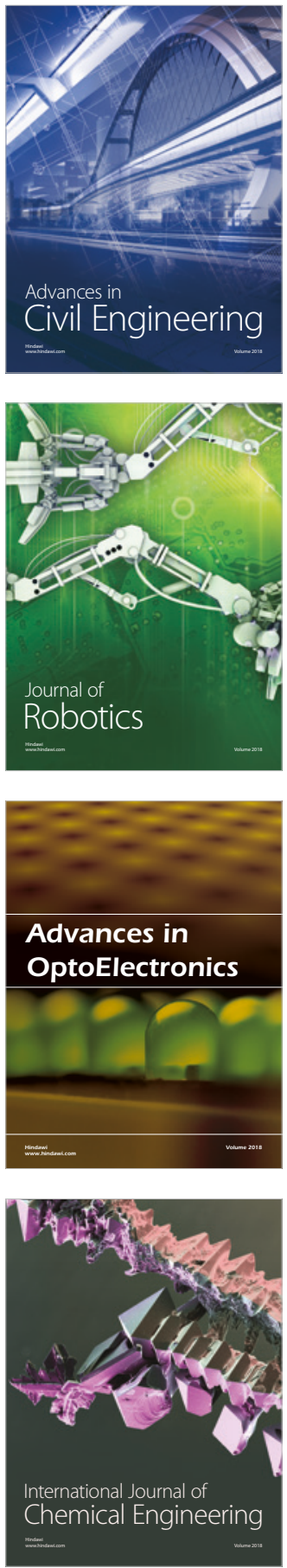

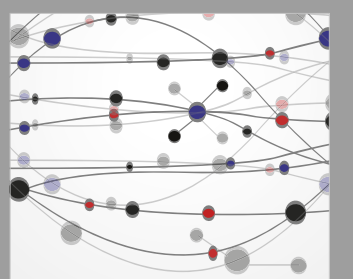

\section{Rotating \\ Machinery}

The Scientific World Journal

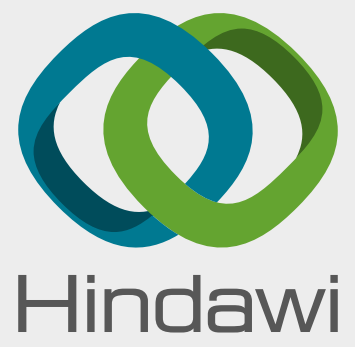

Submit your manuscripts at

www.hindawi.com
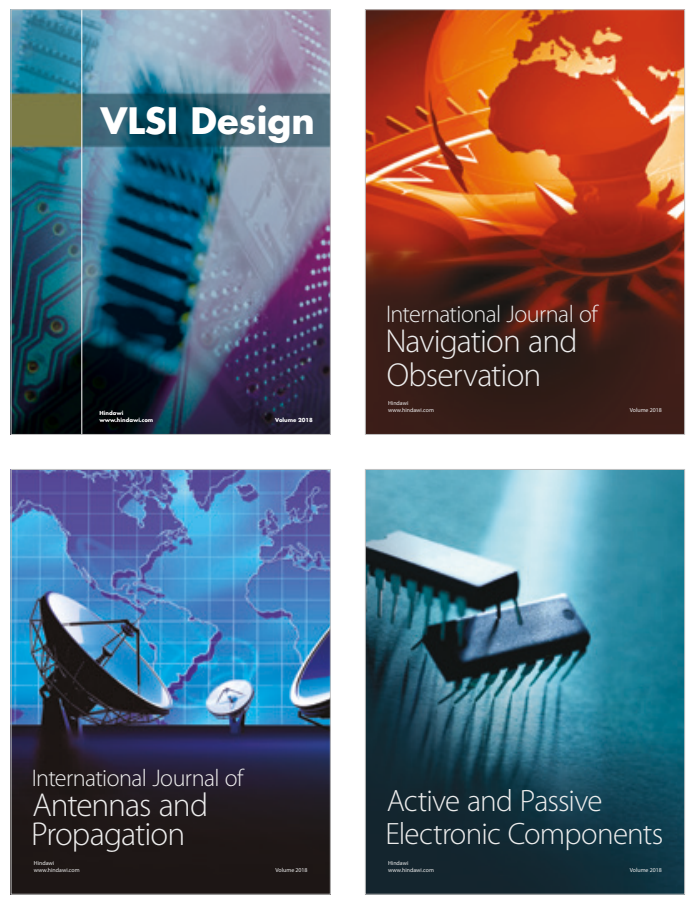
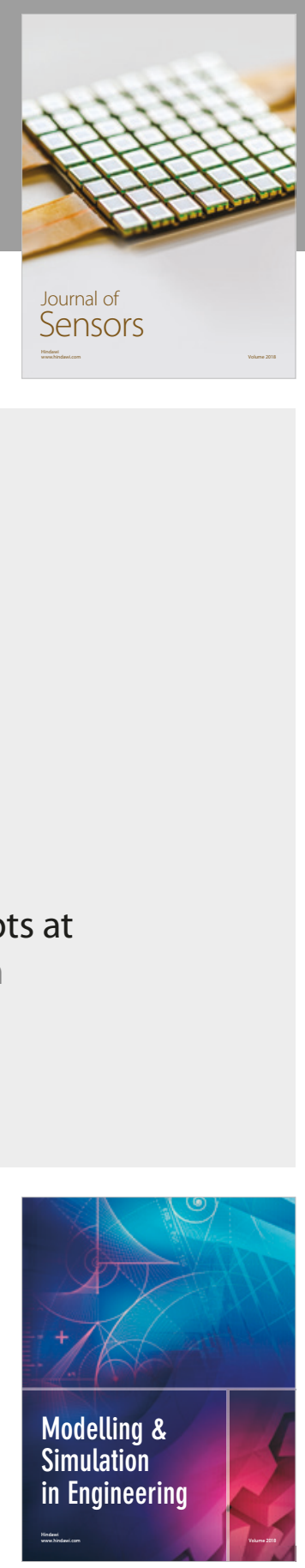

\section{Advances \\ Multimedia}
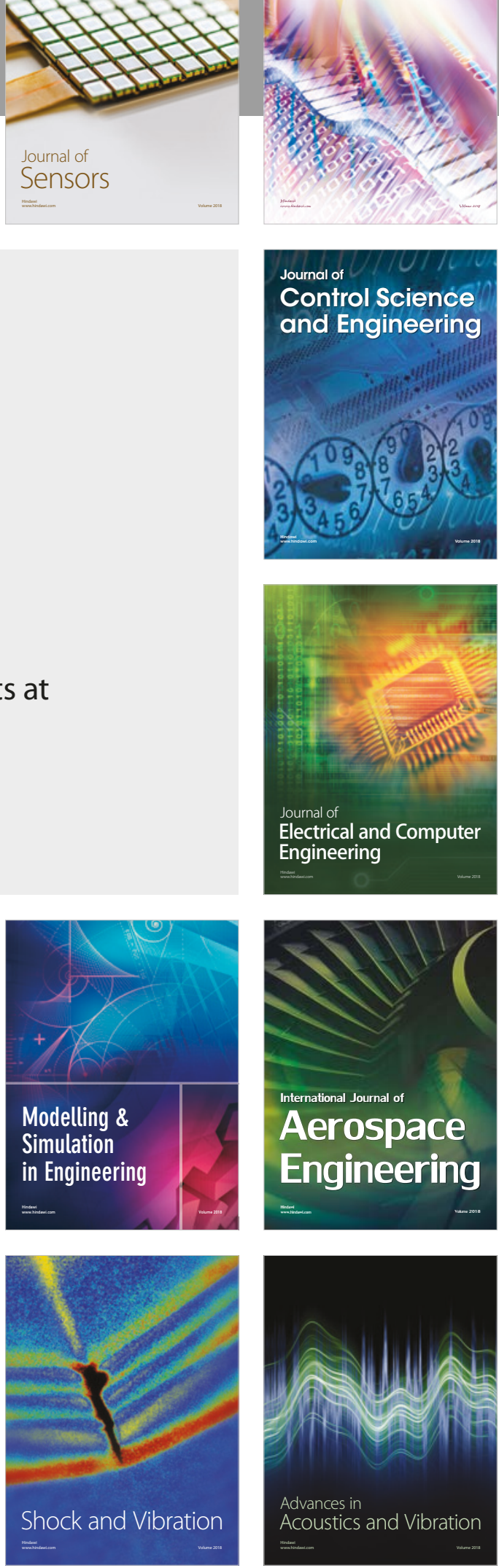